\title{
BMJ Open The multiple lifestyle modification for patients with prehypertension and hypertension patients: a systematic review protocol
}

\author{
Juan Li, ${ }^{1}$ Hui Zheng, ${ }^{1}$ Huai-bin Du, ${ }^{2}$ Xiao-ping Tian, ${ }^{2}$ Yi-jing Jiang, ${ }^{3}$ \\ Shao-lan Zhang, ${ }^{4}$ Yu Kang, ${ }^{5}$ Xiang Li, ${ }^{2}$ Jie Chen, ${ }^{1}$ Chao Lu, ${ }^{1}$ Zhen-hong Lai, ${ }^{1}$ \\ Fan-rong Liang ${ }^{1}$
}

To cite: Li J, Zheng $\mathrm{H}$, Du H-bin, et al. The multiple lifestyle modification for patients with prehypertension and hypertension patients: a systematic review protocol. BMJ Open 2014;4:e004920. doi:10.1136/bmjopen-2014004920

- Prepublication history for this paper is available online. To view these files please visit the journal online (http://dx.doi.org/10.1136/ bmjopen-2014-004920).

$\mathrm{JL}$ and $\mathrm{HZ}$ contributed equally.

Received 1 February 2014 Revised 23 July 2014 Accepted 30 July 2014

CrossMark

For numbered affiliations see end of article.

Correspondence to

Fan-rong Liang;

acuresearch@126.com

\section{ABSTRACT}

Introduction: The objective of this systematic review is to investigate the effectiveness, efficacy and safety of multiple concomitant lifestyle modification therapies for patients with hypertension or prehypertension.

Methods and analysis: Electronic searches will be performed in the Cochrane Library, OVID, EMBASE, etc, along with manual searches in the reference lists of relevant papers found during electronic search. We will identify eligible randomised controlled trials utilising multiple lifestyle modifications to lower blood pressure. The control could be drug therapy, single lifestyle change or no intervention. Changes in systolic blood pressure and diastolic blood pressure constitute primary end points, and secondary end points include the number of patients meeting the office target blood pressure, the number of patients reporting microvascular or macrovascular complications, etc. We will extract descriptive, methodological and efficacy data from identified randomised controlled trials (RCTs). We will calculate the relative risk for proportion of patients with a normal blood pressure in the experimental group. Dichotomous data will be analysed using risk difference and continuous data using weighted mean differences, both with $95 \% \mathrm{Cl}$. We will use the $\chi^{2}$ test and the $\mathrm{I}^{2}$ statistic to assess heterogeneity. We will use the fixed effects model to compute the efficacy unless there is evidence of heterogeneity. If heterogeneity of effect size persists with respect to blood pressure change, further metaregression will be performed within groups. We will examine the potential for publication bias by using a funnel plot.

Dissemination: We will synthesise results from RCTs which provide more precise and accurate information on the effect of multiple lifestyle changes on blood pressure. The results of this review will increase the understanding of multiple lifestyle modifications for patients with hypertension or prehypertension.

Trail registration number: Our protocol is registered on PROSPERO (CRD42013006476), http:// www.crd.your.ac.uk/PROSPERO.

\section{Strengths and limitations of this study}

To the best of our knowledge, this is the first systematic review to investigate the effectiveness, efficacy and safety of multiple lifestyle changes for patients with hypertension.

- The results of this systematic review can provide clinicians with useful clinical information to guide their patient care and increase the understanding of lifestyle modifications, as well as motivate patients with hypertension to adopt and maintain multiple lifestyle changes.

- The limitation of this systematic review is that it may be difficult to retrieve all the multiple lifestyle modifications for patients with hypertension. We will cooperate with the experienced medical librarian to design an appropriate search strategy, in order to ensure a broad search.

\section{BACKGROUND}

Essential hypertension continues to be an important public health challenge worldwide. ${ }^{1}$ Hypertension $(7 \%)$ is the leading cause of death followed by stroke $(16.6 \%)$ and heart failure $(7.3 \%) .^{2}$ It is an independent risk factor affecting the development of cardiovascular disease (myocardial infarction, heart failure and stroke), chronic kidney disease and peripheral vascular disease. ${ }^{2}$ Hypertension affects $29.3 \%$ of the adult population in the USA. ${ }^{3}$ It affects almost $18.8 \%$ of the Chinese population according to the fourth National Nutrition and Health Survey in 2002. ${ }^{4}$ The Joint National Committee (JNC)-7 created a new category of hypertension, 'prehypertension', ${ }^{5}$ which is defined as systolic blood pressure (SBP) of 120-139 mm Hg or diastolic blood pressure (DBP) of $80-89 \mathrm{~mm} \mathrm{Hg}$. Patients in this category are at increased risk for progression to hypertension. Numerous studies emerged 
later to investigate the risk of prehypertension for various types of adverse outcomes, including stroke, coronary heart disease and cardiovascular disease (CVD), and all-cause mortality. ${ }^{6-9}$ From 2005 to 2006, approximately three of eight adults in the USA had blood pressure in the prehypertensive range of 120-139/ $80-89 \mathrm{~mm} \mathrm{Hg}$ and roughly one in eight adults had blood pressure in the range of $130-139 / 85-89 \mathrm{~mm}$ Hg. ${ }^{10}$ Furthermore, the number of patients with hypertension and prehypertension patients is still increasing. Although hypertension and prehypertension affects a large portion of the population, recognition and adequate treatment are less than ideal. ${ }^{11}$ Despite the availability of multiple effective antihypertensive drugs, hypertension control rates remain poor. Hypertension expenditures represent a significant amount of healthcare resource use. ${ }^{12}$ The total annual medical expenditures attributed to hypertension including comorbidities are estimated to range from US $\$ 108$ to US $\$ 110$ billion. ${ }^{13}$ As we all known, some antihypertensive drugs may cause side effects such as dizziness, headache, fatigue, chest discomfort, cough and sexual dysfunction, prompting some patients to discontinue therapy.

Therefore, there is an urgent need to develop and implement non-pharmacological methods (such as salt reduction, weight loss and exercise) to improve prevention and treatment of this major cause of death. As a modifiable risk factor, treatment of prehypertension and hypertension through lifestyle changes is a vital approach. ${ }^{14}$ Lifestyle interventions have a profound impact on the national economic impact of hypertension. ${ }^{12}$ The JNC-8 ${ }^{15}$ and $2013 \mathrm{ESH} / \mathrm{ESC}^{16}$ guidelines and the results of trials ${ }^{17-20}$ on lifestyle modification for hypertension recommended that lifestyle modification is capable of lowering the blood pressure. However, little is known about the efficacy of programmes that intervene on multiple lifestyle factors to maximise the blood pressure lowering effect. Evidence regarding the magnitude of multiple lifestyle modification-related reductions in blood pressure is inconsistent. Also, the benefits of multiple lifestyle changes have not been carefully examined. We raised the following questions: (1) Is a multiple concomitant lifestyle modification therapy effective for patients with hypertension or prehypertension? (2) If so, what is the magnitude of multiple lifestyle modificationrelated reductions on blood pressure? We will pool results from randomised control trials to provide more precise and accurate information on the effect of multiple lifestyle changes on blood pressure.

\section{METHOD AND DESIGN}

\section{Criteria for considering studies for this review \\ Type of studies}

The review will include randomised controlled trials published in the English language only. Completed or ongoing trials will be included in this review, as well as trials using only the parallel design.
Participant characteristics

Participants to be included are adult participants ( $\geq 18$ years of age) presenting with prehypertension (SBP: $120-139 \mathrm{~mm} \mathrm{Hg}$; DBP $80-89 \mathrm{~mm} \mathrm{Hg}$ ) or essential hypertension (SBP $\geq 140 \mathrm{~mm} \mathrm{Hg} ; \quad \mathrm{DBP} \geq 90 \mathrm{~mm} \mathrm{Hg}$ ), according to the JNC guideline. ${ }^{5}$ Restrictions will not be placed on the gender or ethnic background of participants.

\section{Intervention characteristics}

Studies utilising multiple lifestyle modifications (a combination of at least two of the following methods: weight loss, ${ }^{21}$ DASH diet, ${ }^{22-25}$ dietary sodium reduction, ${ }^{26}$ physical activity, ${ }^{27}$ moderation of alcohol consumption) will be reviewed. We include multiple lifestyle modifications consisting of at least dietary pattern changes (including sodium reduction) and physical activity. We also allow cointerventions if they are applied in all arms (including antihypertensive drugs, self-monitoring). The lifestyle modification should last for at least 4 weeks, and the follow-up phase should be at least 4 weeks. And how did the patients implement the lifestyle changing must be specified in the original article. However, studies using nurse, physician or pharmacologist counselling (face-to-face, group counselling, etc), and computer or telephone monitoring will be excluded.

\section{Control arm characteristics}

Studies utilising any type of non-pharmacological treatment, single lifestyle change, waiting list, no intervention or any type of antihypertensive drugs as a control arm will be included.

We will include the following controls:

1. Multiple lifestyle modifications+antihypertensive drugs versus antihypertensive drugs;

2. Multiple lifestyle modifications versus waiting list control or no intervention;

3. Multiple lifestyle modifications versus sham control (irrelevant lifestyle modification for hypertension);

4. Multiple lifestyle modifications versus single lifestyle modification.

\section{Outcome measures}

The primary outcomes of this systematic review are the changes of SBP and DBP between baseline and after treatment. The secondary outcomes including (1) the number of patients meeting the office target blood pressure; (2) the number of patients reporting microvascular complications (retinopathy, neuropathy, nephropathy); (3) the number of patients with macrovascular complications (cardiovascular disease, stroke/transient ischaemic attack, myocardial infarction, peripheral vascular disease); (4) All cause mortality, cardiovascular mortality, cerebrovascular mortality.

The whole process of this systematic review is presented in figure 1. 


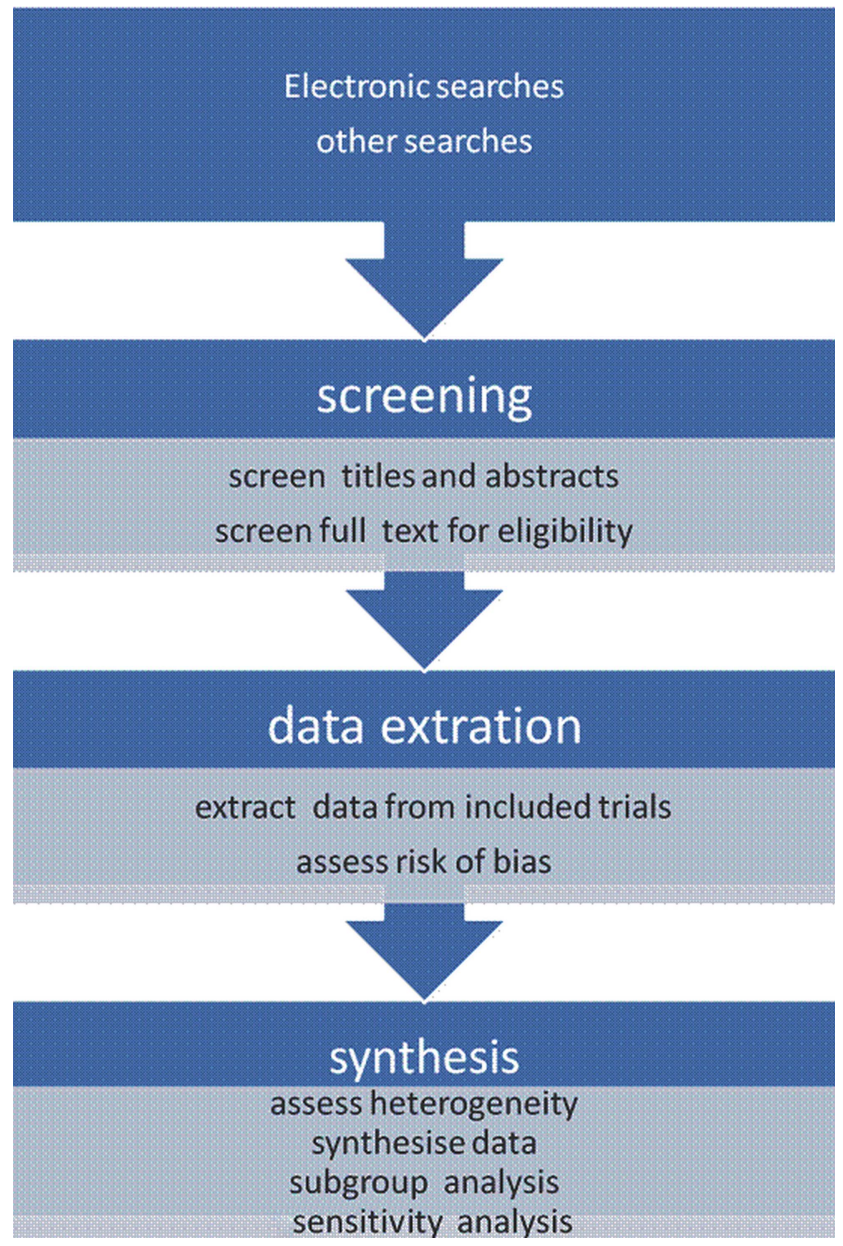

Figure 1 The flow chart of the systematic review.

\section{Database and search}

We will identify relevant randomised controlled trials, only in the English language, by a systematic search of EMBASE, MEDLINE, AMED, the Cochrane library, CINAHL, ISI Web of Knowledge and the Cochrane Central Registry of Controlled Trials. An experienced medical librarian designed terms (see table 1) to retrieve trials enrolling participants with prehypertension or hypertension managed with multiple lifestyle modifications. Reviewers will scan all the retrieved trials and other relevant articles. We will review the reference list of previously published articles for possible candidates. We also searched for ongoing trials from mainstream registries, such as the metaRegister of Controlled Trials, ClinicalTrials.gov trials registry, the Australian New Zealand Clinical Trials Registry, etc.

\section{Studies selection}

Two experienced reviewers (JL and JC) will independently screen the titles and abstracts of identified citations for potential eligible trials and exclude the duplicates. Then they will apply eligibility criteria to the full text of potentially eligible trials. If the reviewers ( $\mathrm{HZ}$ and $\mathrm{JL}$ )
Table 1 Search strategy used in the OVID MEDLINE database

\begin{tabular}{ll}
\hline Number & Search terms \\
\hline 1 & randomised controlled trial.pt. \\
2 & randomized controlled trial.pt. \\
3 & controlled clinical trial.pt. \\
4 & randomized.ab. \\
5 & randomised.ab. \\
6 & placebo.ab. \\
7 & randomly.ab. \\
8 & trial.ab. \\
9 & groups.ab. \\
10 & 1 or 2 or 3 or 4 or 5 or 6 or 7 or 8 or 9 \\
11 & exp hypertension/ \\
12 & exp high blood pressure/ \\
13 & exp prehypertension/ \\
14 & hyperten $\$ . t w, m p$. \\
15 & high blood press $\$ . t w, m p$. \\
16 & prehyperten $\$ . t w, m p$. \\
17 & 11 or 12 or 13 or 14 or 15 or 16 \\
18 & exp life style/ \\
19 & exp lifestyle/ \\
20 & exp lifestyle modification/ \\
21 & exp smoke/ \\
22 & exp diet/ \\
23 & exp weight loss/ \\
24 & exp alchohol consumption/ \\
25 & lifestyle.tw,mp. \\
26 & lifestyle modification.tw,mp. \\
27 & smoke.tw,mp. \\
28 & diet.tw,mp. \\
29 & weight loss.tw,mp. \\
30 & alchohol consumption.tw,mp. \\
31 & lifestyle $\$ . t w, m p$. \\
32 & 18 or 19 or 20 or 21 or 22 or 23 or 24 or 25 or \\
33 & 26 or 27 or 28 or 29 or 30 or 31 \\
&
\end{tabular}

cannot clearly screen the trials according to the titles and abstracts, the other two reviewers (JC and CL) will screen the full copies of these studies. Conflicts will be resolved by team discussion. If disagreements persist, a third reviewer (F-RL) will be consulted.

\section{Data extraction}

Data concerning patient characteristics (age, gender, BMI, etc), study characteristics (interventions, control arms, length of intervention, length of follow-up and so on), type of blood pressure monitoring (office, home, ambulatory), co-intervention and outcome (SBP, DBP, cardiovascular events, etc) were extracted independently by two reviewers (HZ and JL) using a standardised extraction form. If data are missing from published reports, we will contact the authors of the included trials for further information. Disagreements will be resolved by team discussion. FRL will review the data to ensure no input data errors. 


\section{Risk of bias assessment}

Two reviewers will assess the risk of bias independently using the Cochrane risk of bias tool. The following items will be assessed (http://www.cochrane-handbook.org): random sequence generation (selection bias); allocation concealment (selection bias); blinding (performance bias and detection bias); incomplete outcome data (attrition bias); selective outcome reporting (reporting bias). The risk of bias is categorised as a low/unclear/ high risk of bias. Trials which meet all criteria will be judged as having a low risk of bias, whereas trials which meet none of the criteria will be judged as having a high risk of bias and trials with insufficient information to judge will be classified as an unclear risk of bias. Disagreement between the reviewers over the risk of bias in specific studies will be resolved by discussion and consensus, with the involvement of a third review author (FRL) where necessary.

\section{Strategy for data synthesis}

Weight mean differences will be calculated for the overall mean change from baseline of SBP and DBP (using office, casual or ambulatory blood pressure measurements) for experimental and control groups. When the proportion of responders (participants who report a $\mathrm{BP}$ lower than $140 / 90 \mathrm{~mm} \mathrm{Hg}$ after treatment) is reported, we will calculate the relative risk using the control group as a reference.

We will assess heterogeneity using the Higgins $\mathrm{I}^{2}$ statistics; an $\mathrm{I}^{2}>50 \%$ will be considered as heterogeneity existing among studies. In that case, we will first use metaregression to find out the source of heterogeneity (age, gender, interventions, outcome measurements, etc) and then use subgroup analysis. If the problem remains, we will use the random effect model and explain the results with caution. A fixed effect model will be used when there is no evidence of heterogeneity.

We will examine the potential for publication bias by using a funnel plot.

If the data are not appropriate to perform a meta-analysis, we will just do a narrative synthesis.

Analyses will be performed with $\mathrm{R}$ project 3.02 (http://www.r-project.org). If the required data are not reported, we will request data from the corresponding author. However, if the missing data are out of reach, we will exclude such studies and synthesise the data from the rest of the included studies.

\section{Analysis of subgroups or subset}

To investigate the potential heterogeneity across studies, we will conduct subgroup analysis based on the different combinations of lifestyles, classification of blood pressure (prehypertension, mild hypertension, moderate hypertension, severe hypertension) and quality of evidence. Random effects metaregression models will be used to quantify the difference between subgroups and to test for statistical significant interactions among subgroups.
Sensitivity analysis

We will conduct sensitivity analysis to assess the effects of individual studies on the combined estimates and determine whether certain studies dominate the pooled event (particularly if these studies were at a high risk of bias). If certain studies have borderline eligibility status for any reason, analysis will be conducted with and without such studies.

\section{DISCUSSION}

Many existing systematic reviews ${ }^{26}{ }^{27}$ focus on the effect of a single lifestyle modification on blood pressure. Although the single lifestyle modification has been shown to lower elevated blood pressure, it is currently not known whether their combined use produces an additive antihypertensive effect. Of the various lifestyle interventions, physical activity and dietary intervention have been shown to diminish the blood pressure and reduce CVD events, which have emerged as the two most effective and physiologically desirable approaches. That is why we defined that the multiple lifestyle changes should at least include physical activity and dietary intervention.

This is first systematic review that attempts to investigate the effectiveness of multiple lifestyle changes simultaneously. The aim of this systematic review is to synthesise the available evidence of effectiveness of multiple lifestyle interventions in the treatment of prehypertension and hypertension. The systematic review also intends to help clinicians to make decisions on the clinical applicability of multiple lifestyle modifications; at the same time, the results of this review will increase the understanding of multiple lifestyle changes for patients with prehypertension and hypertension; encourage and motivate patients with prehypertension or hypertension to adopt and maintain mulitple lifestyle changes in their daily home life so as to improve hypertension outcomes.

However, we will select the trials which implemented the multiple lifestyle interventions for at least 4 weeks. The multiple lifestyle interventions have to be sustained for a time to achieve the effect of lowering the blood pressure. Trials employing lifestyle modification therapies usually intervene for more than 4 weeks. ${ }^{17} 2028$

The reason why we excluded trials utilising counselling or monitoring was that both of them had been reported to achieve antihypertensive effects. ${ }^{29-31}$ No matter counselling or monitoring, lots of manpower, material resources and financial resources have to be invested. Moreover, we excluded such trials so as to investigate the genuine effects of multiple lifestyle modifications.

This systematic review will provide a general view and evidence of the effects of multiple lifestyle changes on blood pressure. The results of this systematic review will also provide clinicians with useful information to guide their patient care. The finding of the systematic review will be disseminated through publication in a peer- 
reviewed journal; reporting of the systematic review will follow recommendations described in the Preferred Reporting Items for Systematic Reviews and Meta-Analyses (PRISMA) statement and the Cochrane Handbook for Intervention Reviews, and will be formatted according to the specific journal publication guideline.

\section{Author affiliations}

${ }^{1}$ Chengdu University of Traditional Chinese Medicine, Chengdu, Sichuan, People's Republic of China

${ }^{2}$ Third Affiliated Hospital of Chengdu University of Traditional Chinese Medicine, Chengdu, Sichuan, People's Republic of China

${ }^{3}$ Fujian University of Traditional Chinese Medicine, Fuzhou, Fujian, People's Republic of China

${ }^{4}$ Chengdu Medical College, Chengdu, Sichuan, People's Republic of China

${ }^{5}$ First Affiliated Hospital of Chengdu University of Traditional Chinese

Medicine, Chengdu, Sichuan, People's Republic of China

Acknowledgements The author would like to thank Wen-xiang Zeng for his assistance in the development of the search strategies for this protocol.

Contributors $\mathrm{HZ}$ and $\mathrm{JL}$ conceptualised the study and prepared the draft of the research proposal. H-BD, XL, JC, X-PT, Y-JJ, F-RL, S-LZ, YK, CL and $\mathrm{Z}-\mathrm{HL}$ assisted with the manuscript preparation. All authors have read and approved the final manuscript.

Funding This trial was supported financially by the National Basic Research Program of China‘973Program' (No. 2012CB518501).

Patient consent Obtained.

Provenance and peer review Not commissioned; externally peer reviewed.

Data sharing statement The additional unpublished data are still being extracted by $\mathrm{JL}$ and $\mathrm{HZ}$ and will be published later.

Competing interests None.

Open Access This is an Open Access article distributed in accordance with the Creative Commons Attribution Non Commercial (CC BY-NC 3.0) license, which permits others to distribute, remix, adapt, build upon this work noncommercially, and license their derivative works on different terms, provided the original work is properly cited and the use is non-commercial. See: http:// creativecommons.org/licenses/by-nc/3.0/

\section{REFERENCES}

1. Kearney PM, Whelton M, Reynolds K, et al. Global burden of hypertension: analysis of worldwide data. Lancet 2005;365:217-23.

2. Lloyd-Jones D, Adams RJ, Brown TM, et al. Heart disease and stroke statistics-2010 update: a report from the American Heart Association. Circulation 2010;121:e46-215.

3. Ong KL, Cheung BM, Man YB, et al. Prevalence, awareness, treatment, and control of hypertension among United States adults 1999-2004. Hypertension 2007;49:69-75.

4. Li L, Rao K, Kong L, et al. [A description on the Chinese national nutrition and health survey in 2002]. Zhonghua Liu Xing Bing Xue Za Zhi 2005;26:478-84.

5. Chobanian AV, Bakris GL, Black HR, et al. Seventh report of the joint national committee on prevention, detection, evaluation, and treatment of high blood pressure. Hypertension 2003;42:1206-52.

6. Asayama K, Ohkubo T, Kikuya M, et al. Prediction of stroke by self-measurement of blood pressure at home versus casual screening blood pressure measurement in relation to the Joint National Committee 7 Classification The Ohasama Study. Stroke 2004;35:2356-61.

7. Carlsson AC, Theobald H, Hellénius M-L, et al. Cardiovascular and total mortality in men and women with different blood pressure levels-A 26-year follow-up. Blood Press 2009;18:105-10.
8. Pimenta E, Oparil S. Prehypertension: epidemiology, consequences and treatment. Nat Rev Nephrol 2009;6:21-30.

9. Vasan RS, Larson MG, Leip EP, et al. Impact of high-normal blood pressure on the risk of cardiovascular disease. $N$ Engl J Med 2001;345:1291-7

10. Ostchega Y, Yoon SS, Hughes J, et al. Hypertension awareness, treatment, and control-continued disparities in adults: United States, 2005-2006. NCHS Data Brief 2008:1-8.

11. Chow CK, Teo KK, Rangarajan S, et al. Prevalence, awareness, treatment, and control of hypertension in rural and urban communities in high-, middle-, and low-income countries. JAMA 2013;310:959-68.

12. Balu S, Thomas J III. Incremental expenditure of treating hypertension in the United States. Am J Hypertens 2006;19:810-16.

13. Hodgson TA, Cai L. Medical care expenditures for hypertension, its complications, and its comorbidities. Medical Care 2001;39:599-615.

14. Meng X-J, Dong G-H, Wang D, et al. Epidemiology of prehypertension and associated risk factors in urban adults from 33 communities in China-the CHPSNE study. Circ J 2012:76:900.

15. James PA, Oparil S, Carter BL, et al. 2014 evidence-based guideline for the management of high blood pressure in adults: report from the panel members appointed to the Eighth Joint National Committee (JNC 8). JAMA 2014;311:507-20.

16. Taylor J. 2013 ESH/ESC guidelines for the management of arterial hypertension. Eur Heart $J$ 2013;34:2108-9.

17. Appel LJ, Champagne CM, Harsha DW, et al. Effects of comprehensive lifestyle modification on blood pressure control: main results of the PREMIER clinical trial. JAMA 2003;289:2083-93.

18. Dusek JA, Hibberd PL, Buczynski B, et al. Stress management versus lifestyle modification on systolic hypertension and medication elimination: a randomized trial. $J$ Altern Complement Med 2008;14:129-38.

19. Elmer PJ, Obarzanek E, Vollmer WM, et al. Effects of comprehensive lifestyle modification on diet, weight, physical fitness and blood pressure control: 18-month results of a randomized trial. Ann Intern Med 2006;144:485-95.

20. Miller ER, Erlinger TP, Young DR, et al. Results of the diet, exercise, and weight loss intervention trial (DEW-IT). Hypertension 2002;40:612-18.

21. Aucott L, Poobalan A, Smith WCS, et al. Effects of weight loss in overweight/obese individuals and long-term hypertension outcomes: a systematic review. Hypertension 2005;45:1035-41.

22. Appel LJ, Moore TJ, Obarzanek E, et al. A clinical trial of the effects of dietary patterns on blood pressure. $N$ Engl J Med 1997;336:1117-24.

23. Moore TJ, Conlin PR, Ard J, et al. DASH (Dietary Approaches to Stop Hypertension) diet is effective treatment for stage 1 isolated systolic hypertension. Hypertension 2001;38:155-58.

24. Earl GL, Henstenburg JA. Dietary approaches to hypertension: a call to pharmacists to promote lifestyle changes. J Am Pharm Assoc 2012:52:637-45.

25. Sacks FM, Campos H. Dietary therapy in hypertension. $N$ Engl J Med 2010;362:2102-12.

26. He F, MacGregor G, Correspondence G. Effect of modest salt reduction on blood pressure: a meta-analysis of randomized trials. Implications for public health. J Hum Hyperten 2002;16:761-70.

27. Whelton SP, Chin A, Xin X, et al. Effect of aerobic exercise on blood pressurea meta-analysis of randomized, controlled trials. Ann Intern Med 2002:136:493-503.

28. Elmer P, Obarzanek E, Vollmer W, et al. PREMIER Collaborative Research Group: effects of comprehensive lifestyle modification on diet, weight, physical fitness, and blood pressure control: 18-month results of a randomized trial. Ann Intern Med 2006;144:485-95.

29. Kastarinen MJ, Puska PM, Korhonen MH, et al. Non-pharmacological treatment of hypertension in primary health care: a 2-year open randomized controlled trial of lifestyle intervention against hypertension in eastern Finland. J Hypertens 2002;20:2505-12.

30. Miura S-i, Yamaguchi $\mathrm{Y}$, Urata $\mathrm{H}$, et al. Efficacy of a multicomponen program (patient-centered assessment and counseling for exercise plus nutrition [PACE+ Japan]) for lifestyle modification in patients with essential hypertension. Hypertens Res 2004;27:859-64.

31. Svetkey LP, Pollak KI, Yancy WS, et al. Hypertension Improvement Project Randomized Trial of quality improvement for physicians and lifestyle modification for patients. Hypertension 2009;54:1226-33. 